\title{
Chaotic dynamics in semiconductor lasers subjected to polarization-rotated optical feedback
}

\author{
Yasutoshi Takeuchi, ${ }^{1}$ Rui Shogenji, ${ }^{2}$ and Junji Ohtsubo ${ }^{2, a)}$ \\ ${ }^{1}$ Graduate School of Engineering, Shizuoka University, 3-5-1 Johoku, Naka-ku, \\ Hamamatsu 432-8561, Japan \\ ${ }^{2}$ Faculty of Engineering, Shizuoka University, 3-5-1 Johoku, Naka-ku, Hamamatsu 432-8561, Japan
}

(Received 2 September 2008; accepted 17 October 2008; published online 4 November 2008)

\begin{abstract}
In-phase or synchronized oscillations between transverse electric (TE) and transverse magnetic (TM) modes with a loop delay are common phenomena in the numerical studies of semiconductor lasers subjected to polarization-rotated optical feedback. However, we experimentally find antiphase oscillations of laser output powers with zero time lag under appropriate conditions of orthogonal polarization optical feedback. Antiphase oscillations are well reproduced by the numerical simulations for the experimental model taking into account the frequency detuning between the two modes. (C) 2008 American Institute of Physics. [DOI: 10.1063/1.3020302]
\end{abstract}

Chaotic dynamics in semiconductor lasers with optical feedback have been extensively studied for those two decades. ${ }^{1}$ The system generates very fast chaotic signals and it is used as transmitter and receiver in chaotic secure communications since we can construct a very high-speed communication channel. ${ }^{2}$ In usual, an edge-emitting semiconductor laser oscillates at transverse-electric mode (TE mode) and TE optical feedback is used in those studies. Another scheme is polarization-rotated optical feedback from TE mode to transverse magnetic mode (TM mode) ${ }^{3-8}$ Recently, chaos synchronization based on the scheme of orthogonal TE-TM feedback and coupling is proposed to avoid locking of optical carrier frequency between transmitter and receiver lasers after transmission of a chaotic carrier over long distance in chaotic secure communications. ${ }^{4,7}$ However, a few studies have been presented for the dynamics of TE-TM feedback and the dynamics have not been fully understood yet.

The dynamics of polarization-rotated optical feedback is quite different from those of ordinary optical feedback. Gavrielides et al. studied differences of the dynamics between TE-TE and TE-TM optical feedback in semiconductor lasers. ${ }^{3}$ According to their study, to excite chaotic TM-mode oscillations in a semiconductor laser, one requires a strong optical feedback to the laser. There is no threshold reduction in the case of polarization-rotated optical feedback and the laser oscillation starts at the same bias injection current as that of the solitary laser. Furthermore, the slope efficiency in the $L-I$ characteristics of TE mode almost remains the same as that of the solitary laser. It is noted that the temporal waveform of the TM mode is delayed with respect to that of the TE mode by the propagation time $\tau$ of the external feedback loop, showing that the TM mode follows the delayed feedback signal (in-phase oscillations). These results mean that the interaction between the TE- and TM-mode intensities through the carrier density generates chaotic intensities and the dynamics of both the amplitude and phase are governed by the dynamics of the carrier density.

In this letter, we present different dynamics from the previous results in semiconductor lasers with polarizationrotated optical feedback. The chaotic waveform of TM mode

\footnotetext{
${ }^{\text {a) }}$ Author to whom correspondence should be addressed. Electronic mail: tajohts@ipc.shizuoka.ac.jp.
}

resembles to that of TE mode; however, the TM mode oscillates with antiphase manner to the TE mode in the present experiment. The observed time delay between the two modes is zero. Therefore, the origin of the dynamics is different from the previous ones based on the optical injection and locking from TE to TM mode. ${ }^{5,6,8}$ First, we present experimental results and, after that, we show the numerical simulations using the rate equations.

To realize orthogonal polarization optical feedback, two schemes are commonly used; one is a ring-loop model, by which we can avoid multiple-reflection scheme within the optical feedback loop. ${ }^{5,7}$ The TE mode from a semiconductor laser goes through a polarization beam splitter and is converted to a TM mode by $\lambda / 4$ and $\lambda / 2$ waveplates. Another example of orthogonal polarization feedback is the use of a Faraday rotator together with a polarizer. ${ }^{6,8}$ The TE polarized beam enters a Faraday rotator whose input polarizer is removed and the beam's polarization rotates $45^{\circ}$. The beam reflected by the feedback mirror is reinjected to the rotator and this creates an orthogonal polarized beam to the laser oscillation mode (i.e., TM mode). In this configuration, the reflected vertically polarized beam from the laser facet is once passed through the rotator, but it is blocked by the polarizer. Thus, a single feedback loop is also guaranteed in this setup. For the both systems, the effect of orthogonal polarization feedback can be described by the same rate equations. We employed the latter scheme in the experiment.

Figure 1 shows the experimental setup. The semiconductor laser used in the experiment was a single-mode multiquantum-well laser (Hitachi HL7851G) that oscillated at a wavelength of $780 \mathrm{~nm}$ and a maximum power of $50 \mathrm{~mW}$. The threshold of the laser was $41.0 \mathrm{~mA}$ at the temperature of $25.0^{\circ} \mathrm{C}$. The laser mainly oscillated at a single TE mode under the operating condition and the orthogonal TM mode was scarcely detectable at the solitary oscillation. The laser was biased at $100 \mathrm{~mA}$. Under this operating condition, the relaxation oscillation frequency, which plays an important role for the laser dynamics, was about $4.5 \mathrm{GHz}$. In the experimental setup, the TE-mode beam was collimated by a lens (CL) and passes through a beam splitter (BS). The beam entered a Faraday rotator (ROT), by which the polarization of the beam was rotated by $45^{\circ}$. A polarizer (PL) whose polarization direction was coincident with the rotation was 


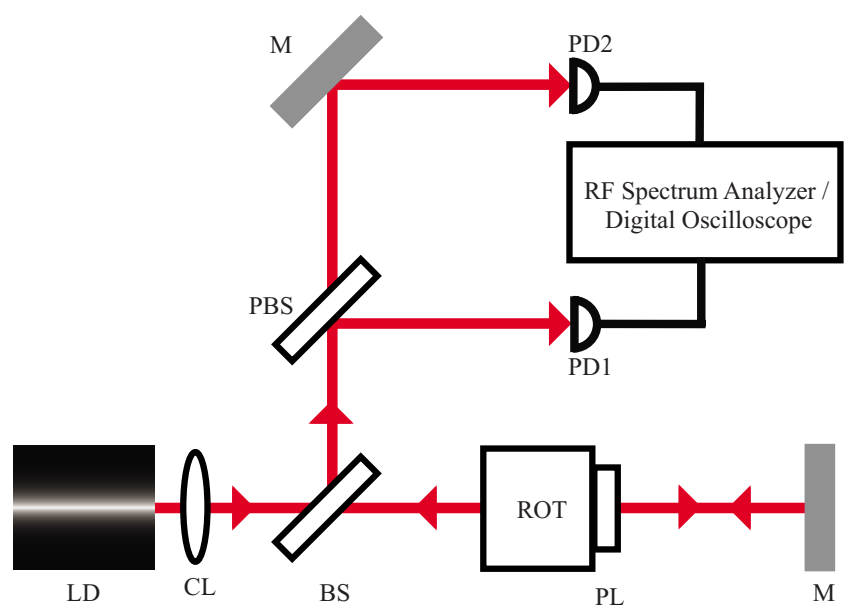

FIG. 1. (Color online) Experimental setup. LD is laser diode, CL is collimating lens, BS is beam splitter, ROT is Faraday rotator, PL is polarizer, M is mirror, $\mathrm{PBS}$ is polarizing beam splitter, and PD1, 2 are photodetectors.

placed at the exit face of the Faraday rotator. The beam was reflected by a mirror (M) and was fed back to the laser. The feedback beam was again rotated by $45^{\circ}$ through the Faraday rotator. Thus the TM-mode feedback to the laser was realized. The external cavity length was $45 \mathrm{~cm}$, which corresponded to the feedback time of $3.0 \mathrm{~ns}$. The other beam reflected by the beam splitter was introduced to a polarization beam splitter (PBS), by which TE and TM beams were separated and fed into photodetectors (PDs) (NEW FOCUS 1554-50: bandwidth of $12 \mathrm{GHz})$. Then the two beams were analyzed by a rf spectrum analyzer (HP 8595E: bandwidth of $6.5 \mathrm{GHz}$ ) and a digital oscilloscope (HP 54845A: bandwidth of $1.5 \mathrm{GHz}$ ). Also the laser oscillations were monitored by an optical spectrum analyzer (ADVANTEST Q8344A: maximum resolution of $0.05 \mathrm{~nm}$ ) and a Fabry-Pérot spectrometer (COHERENT Model 240: free spectral range of $7.5 \mathrm{GHz}$ ).

Figure 2 shows the experimental results of TE- and TMmode oscillations. The TE- and TM-mode waveforms are shown in Fig. 2(a). The external intensity feedback ratio
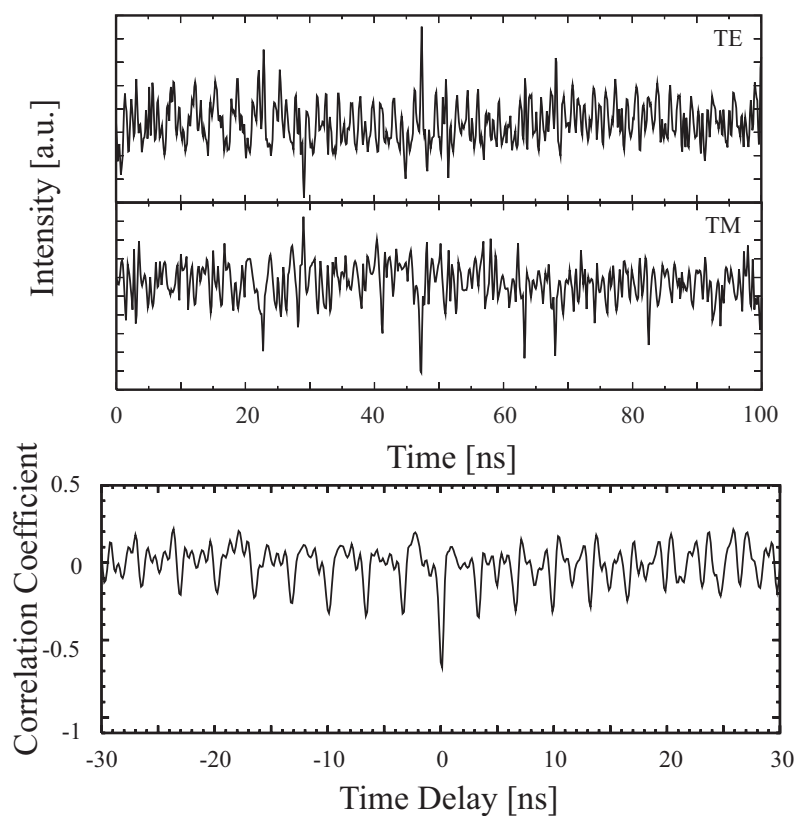

FIG. 2. Experimental results. (a) Time series for TE and TM modes and (b) correlation plot. from TE to TM optical power counted in the external loop was $13 \%$. Under this feedback condition, the power ratio between TE- and TM-mode oscillations was 1000:7. The frequency detuning between the TE mode and the excited TM mode was $-700 \mathrm{MHz}$. Although the difference is small, this nonzero frequency detuning plays an important role as shown in the theoretical discussion. Since the $3 \mathrm{~dB}$ bandwidth of the digital oscilloscope was $1.5 \mathrm{GHz}$, the main chaotic fluctuations (corresponding to the relaxation oscillation frequency of $4.5 \mathrm{GHz}$ ) were not observable and the observed waveforms were low-pass filtered ones. As easily seen from the two waveforms, the TM output power oscillates at antiphase manner for the TE output power and the time shift of the antiphase oscillation is zero. The antiphase oscillation is clearly displayed by the correlation plot between the two waveforms. The correlation coefficient is -0.68 . The small periodic peaks in the correlation plot correspond to a time shift for the external optical feedback loop ( $3 \mathrm{~ns})$. In the pervious studies of a semiconductor laser with orthogonal polarization-rotated optical feedback, ${ }^{5,6}$ a TM mode showed an in-phase oscillation with a TE mode and the TM-mode oscillation was delayed to the TE mode with the loop delay. Therefore, the origin of the phenomenon observed here is different from that of the previous ones.

By employing the rate equations for the experimental model, we have also conducted numerical simulations. Since the feedback ratio from TE to TM mode is strong enough, we must use a model of coherent optical feedback for the complex optical amplitude $E$ and the carrier density $n$ as follows: ${ }^{1}$

$$
\begin{aligned}
\frac{d E_{\mathrm{TE}}(t)}{d t}= & \frac{1}{2}(1-i \alpha) G_{n, \mathrm{TE}}\left\{n(t)-n_{\mathrm{th}, \mathrm{TE}}\right\} E_{\mathrm{TE}}(t) \\
\frac{d E_{\mathrm{TM}}(t)}{d t}= & \frac{1}{2}(1-i \alpha) G_{n, \mathrm{TM}}\left\{n(t)-n_{\mathrm{th}, \mathrm{TM}}\right\} E_{\mathrm{TM}}(t) \\
& +\frac{\kappa}{\tau_{\mathrm{in}}} E_{\mathrm{TE}}(t-\tau) \exp \left[i \left\{-\Delta \omega t+\omega_{\mathrm{TE}} \tau+\phi_{\mathrm{TM}}(t)\right.\right. \\
& \left.\left.-\phi_{\mathrm{TE}}(t-\tau)\right\}\right], \\
\frac{d n(t)}{d t}= & \frac{J}{e d}-\frac{n(t)}{\tau_{s}}-\left\{n(t)-n_{0}\right\}\left\{G_{n, \mathrm{TE}}\left|E_{\mathrm{TE}}(t)\right|^{2}\right. \\
& \left.+G_{n, \mathrm{TM}}\left|E_{\mathrm{TM}}(t)\right|^{2}\right\},
\end{aligned}
$$

where the subscripts TE and TM represent the variables and parameters for TE and TM modes. The gain $G_{n}$ and the carrier density at threshold $n_{\text {th }}$ usually have different values for the TE and TM modes. $\alpha, \kappa$, and $\tau_{\text {in }}$, are the linewidth enhancement factor, the feedback coefficient, and the round trip time within the internal cavity, respectively. $\Delta \omega=\omega_{\mathrm{TE}}-\omega_{\mathrm{TM}}$ is the angular frequency detuning between the two polarization modes $\left(\omega_{\mathrm{TE}}\right.$ and $\omega_{\mathrm{TM}}$ being the laser angular frequencies for the TE and TM modes). $\phi$ is the phase of the laser oscillation. $\tau_{s}$ and $n_{0}$ are the carrier lifetime and the carrier number at transparency. $e$ and $d$ are the elemental charge and the thickness of the active layer, respectively.

The difference in the gains for the TE and TM modes plays an important role to reproduce antiphase oscillations. To realize numerically antiphase oscillations for the two polarization modes, the values of the gains for the TE and TM modes are carefully selected. ${ }^{5-7}$ In the following numerical 

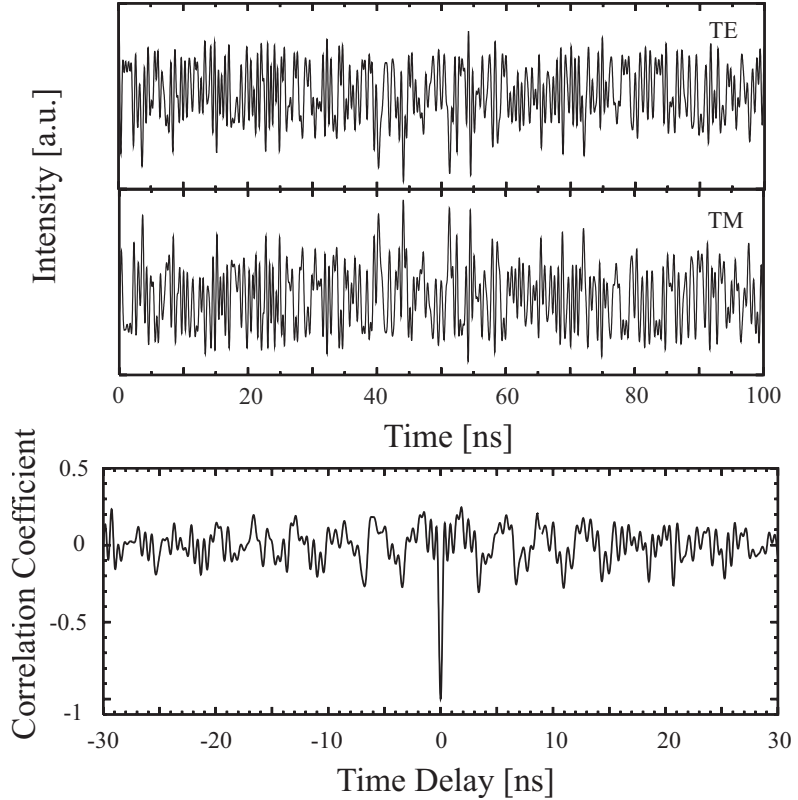

FIG. 3. Numerical results. (a) Time series for TE and TM modes and (b) correlation plot.

simulations, the gains of the TE and TM modes are set as $0.610 \times 10^{-12}$ and $0.530 \times 10^{-12} \mathrm{~m}^{3} \mathrm{~s}^{-1}$, respectively. The threshold carrier densities are assumed to have the same value for the two polarization modes. The same frequency detuning of $-700 \mathrm{MHz}$ as observed in the experiment is assumed. Other parameter values are the photon lifetime of $\tau_{\mathrm{ph}}=1.927 \times 10^{-12} \mathrm{~s}$, the carrier lifetime of $\tau_{s}=2.040$ $\times 10^{-9} \mathrm{~s}$, the feedback ratio of $\kappa / \tau_{\text {in }}=0.010 \times 10^{12} \mathrm{~s}^{-1}$ (corresponding external intensity reflectivity of $0.41 \%$ ), and the linewidth enhancement factor of $\alpha=3.00$. The feedback ratio is compatible with that of the experiment taking into account the losses of the beam intensity fed back into the active layer. The laser is biased at $2.5 J_{\text {th }}$ and the relaxation oscillation frequency at this injection current is $5.06 \mathrm{GHz}$. To compare the experimental data, we used a low-pass filter for the simulated waveforms. The bandwidth of the square low-pass filter was $2 \mathrm{GHz}$. Figure 3 shows the numerical results. TE- and TM-mode oscillations are plotted in Fig. 3(a). The two waveforms show antiphase oscillations with zero time delay. Figure 3(b) is the corresponding correlation plot. The value of the correlation coefficient is -0.99 . We can also observe small periodic peaks in the correlation plot corresponding to the time delay for the external optical feedback loop of $3 \mathrm{~ns}$.

In the numerical simulations, we tested several sets of parameter values for semiconductor lasers and external feedback conditions to reproduce antiphase oscillations in orthogonal polarization-rotated optical feedback. As results, antiphase oscillations were observed for various combinations of the parameter values. In the numerical simulation in Fig. 3, the parameters were almost compatible with the experimental results in Fig. 2. To excite chaotic oscillations for the lower gain mode, a small difference in the gains between the two polarization modes is essential, but the allowance is not so severe. The most important parameter is the frequency detuning. For simulations at zero detuning, we can even reproduce antiphase oscillations, however they are very unstable and limited to a very narrow range of the parameter sets. In usual, stable in-phase oscillations between the two modes are observed at zero frequency detuning. ${ }^{5,7}$ On the other hand, we can easily reproduce antiphase oscillations for a wide range of the parameter values in the presence of frequency detuning, as shown in Fig. 3 (for the case of zero frequency detuning, in-phase time-delayed oscillations can be found, for example, in Fig. 9 of Ref. 5).

The physical interpretation of the zero-lag antiphase dynamics is not clear at present. However, the phenomenon may be considered not as the injection locking effect from TE- to TM-mode optical feedback and injection, but as the effect of predation of light powers between TE- and TMmode oscillations. Indeed, in-phase oscillations are numerically reproduced under the assumption of zero frequency detuning between the two modes ${ }^{5,7}$ and the in-phase dynamics may be caused by optical injection locking. The other important parameter is the feedback fraction. In the previous numerical simulations, ${ }^{5-7}$ very strong optical feedback was assumed (for example, $\kappa / \tau_{\text {in }}=0.125 \times 10^{12} \mathrm{~s}^{-1}$ was used in Ref. 7). In the present study, the strength of orthogonal optical feedback is still strong enough to excite the counterpart polarization mode, but the level is much less than the previous numerical examples. In the previous studies of the numerical simulations, the TM-mode gain is very small compared with the TE-mode gain. Then the TM mode is optically injected by the TE mode and the TM mode shows in-phase oscillations with loop delay. On the other hand, the TE-mode power is wasted and the TM mode receives the power when the difference of the gains becomes small. Another important point is the balance between the two terms in the right hand side of Eq. (2). When the fist term related to the TM mode and the feedback term compete with each other, the two modes tend to oscillate at antiphase manner. As a result, the TE and TM modes show antiphase oscillations with zero time delay.

As a summary, we have observed antiphase oscillations with zero delay in semiconductor lasers subjected to polarization-rotated optical feedback. In the study, the analogue bandwidth of the digital oscilloscope was limited to 1.5 GHz. Therefore, the observed waveforms were the lowpass filtered ones and the full oscillation component including the main chaotic frequency, which corresponds to the laser relaxation oscillation, was not presented. By the preliminary numerical simulations, antiphase dynamics are fairly dependent on the frequency range of the chaotic signals and the antiphase dynamics tend to become obscured for the increase of the covering frequency range. We are now conducting further experimental and numerical investigations for the TE-TM mode-coupled dynamics including the higher frequency bandwidth and the results will be reported elsewhere.

${ }^{1}$ J. Ohtsubo, Semiconductor Lasers: Stability, Instability and Chaos, 2nd ed. (Springer, Berlin, 2007).

${ }^{2}$ J. Ohtsubo, IEEE J. Quantum Electron. 38, 1141 (2002).

${ }^{3}$ A. Gavrielides, T. Erneux, D. W. Sukow, G. Burner, T. McLachlan, J. Miller, and J. Amonette, Opt. Lett. 31, 2006 (2006).

${ }^{4}$ D. W. Sukow, K. L. Blackburn, A. R. Spain, K. J. Babcock, J. V. Bennett, and A. Gavrielides, Opt. Lett. 29, 2393 (2004).

${ }^{5}$ T. Heil, A. Uchida, P. Davis, and T. Aida, Phys. Rev. A 68, 033811 (2003).

${ }^{6}$ D. W. Sukow, A. Gavrielides, T. Erneux, M. J. Baracco, Z. A. Parmenter, and K. L. Blackburn, Phys. Rev. A 72, 043818 (2005).

${ }^{7}$ N. Shibasaki, A. Uchida, S. Yoshimori, and P. Davis, IEEE J. Quantum Electron. 42, 342 (2006)

${ }^{8}$ D. W. Sukow, A. Gavrielides, T. McLachlan, G. Burner, J. Amonette, and J. Miller, Phys. Rev. A 74, 023812 (2006). 